\title{
Adaptive Acquisition and Tracking for Deep Space Array Feed Antennas
}

\author{
Ryan Mukai, Victor A. Vilnrotter, Senior Member, IEEE, Payman Arabshahi, Member, IEEE, and \\ Vahraz Jamnejad, Senior Member, IEEE
}

\begin{abstract}
The use of radial basis function (RBF) networks and least squares algorithms for acquisition and fine tracking of NASA's 70-m-deep space network antennas is described and evaluated. We demonstrate that such a network, trained using the computationally efficient orthogonal least squares algorithm and working in conjunction with an array feed compensation system, can point a 70-m-deep space antenna with root mean square (rms) errors of 0.1-0.5 millidegrees (mdeg) under a wide range of signal-to-noise ratios and antenna elevations. This pointing accuracy is significantly better than the 0.8 mdeg benchmark for communications at Ka-band frequencies $(32 \mathrm{GHz})$. Continuous adaptation strategies for the RBF network were also implemented to compensate for antenna aging, thermal gradients, and other factors leading to time-varying changes in the antenna structure, resulting in dramatic improvements in system performance. The systems described here are currently in testing phases at NASA's Goldstone Deep Space Network (DSN) and were evaluated using Ka-band telemetry from the Cassini spacecraft.
\end{abstract}

Index Terms-Adaptive, antennas, array feed, deep space network, NASA, neural networks, orthogonal least-squares, radial basis function (RBF) networks.

\section{INTRODUCTION}

$\mathbf{T}$ HE NASA Deep Space Network (DSN) is an international network of steerable high-gain reflector antennas, which supports interplanetary spacecraft missions, radio and radar astronomy observations for the exploration of the solar system, and select Earth-orbiting missions. The DSN currently consists of three deep-space communication facilities, placed approximately $120^{\circ}$ apart around the world; at Goldstone, in California's Mojave Desert; near Madrid, Spain; and near Canberra, Australia. This strategic placement permits constant observation of spacecraft as the Earth rotates, and helps make the DSN the largest and most sensitive radio science and telecommunications system in the world.

Over the past years, there has been increasing interest in the use of shorter carrier wavelengths to enhance the DSNs telecommunications and radio science capabilities. Shorter carrier wavelengths, or equivalently higher carrier frequencies, yield greater antenna gains and increased useful bandwidth, with reduced sensitivity to deep-space plasma effects, that tend to degrade the quality of the received signal.

However, there are also new problems associated with the use of higher carrier frequencies, namely greater losses due to

Manuscript received December 4, 2000; revised July 16, 2001

The authors are with the Jet Propulsion Laboratory, California Institute of Technology, Pasadena, CA 91109 USA (e-mail: payman@jpl.nasa.gov).

Publisher Item Identifier S 1045-9227(02)01801-5. gravity-induced antenna deformations and wind, greater sensitivity to misalignments of the radio-frequency (RF) components, and more stringent pointing requirements-all of which are further complicated by time-varying distortions imposed on the antenna structure. Even in the absence of external disturbances, such as wind, time- and elevation-dependent loss components are introduced by gravity as the antenna tracks the target (whether it is a spacecraft or a radio-source). The combination of these factors can lead to unacceptably large pointing errors and signal-to-noise-ratio (SNR) losses if left uncorrected.

Recovery of SNR losses due to gravitational deformation has been addressed in [1]-[3]. Here, we consider the problem of acquiring and tracking spacecraft with sufficient accuracy to maintain acceptably small pointing losses (nominally $0.1 \mathrm{~dB}$ ) on large DSN antennas.

\section{A. Array Feed Compensation System}

A recently developed approach for recovering losses due to gravitational deformations, thermal distortion and wind consists of a real-time compensation system employing a seven-element array of feeds in the focal plane of the antenna's subreflector [1]. The array feed compensation system (AFCS) has been evaluated at the DSN's Goldstone complex, and has successfully demonstrated real-time gravity-compensation and closed-loop tracking of spacecraft and radio-source signals at Ka-band frequencies (nominally $32 \mathrm{GHz}$ ). Its application to recovering losses due to mechanical antenna distortions at high frequencies $(32 \mathrm{GHz}$ or higher) is described in [2] and [3].

A conceptual block diagram of the Ka-band AFCS designed for the DSN's 70-m antennas is shown in Fig. 1. Its main components are an array of seven $22 \mathrm{dBi}$ horns with a separate Ka-band low-noise amplifier (LNA) connected to each horn; a sevenchannel downconverter assembly that converts the $32 \mathrm{GHz} \mathrm{RF}$ signal to $300 \mathrm{MHz}$ IF (intermediate frequency), followed by a seven-channel baseband downconverter assembly that generates 14 real (seven complex) baseband signals. A digital signal processing assembly then extracts parameters from the digital samples in real-time to obtain the optimum combining weights and determine the antenna pointing updates needed to maximize the combined SNR.

In the absence of antenna distortions, a single properly designed receiving horn collects virtually all of the focused signal power. Distortions generally lead to a shift in the peak of the signal distribution, as well as a redistribution of the signal power in the focal plane. This leads to loss of power in the central channel, which can be recovered by the outer horns of an array placed in the focal plane. When the horn signals are multiplied 


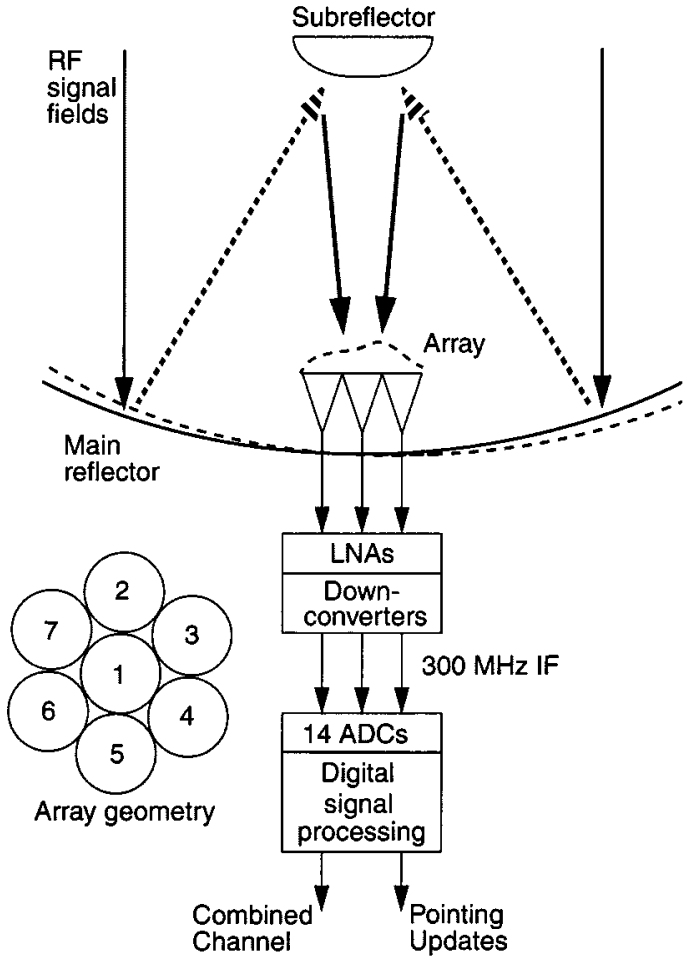

Fig. 1. Conceptual block diagram of the array feed compensation system.

by complex combining weights matched to the instantaneous magnitude and phase of the signal in each channel, the SNR of the combined channel can be improved, approaching that of an undistorted antenna under ideal conditions.

Distortions also affect the pointing of the antenna by introducing shifts in the signal peak. Antenna pointing errors can degrade the received SNR of both single horn and array receivers, particularly at Ka-band or higher frequencies. We shall demonstrate that properly designed neural network and least-squares algorithms effectively remove the time-varying pointing errors, and keep the antenna pointed in the direction of maximum SNR even in the presence of significant antenna distortions.

\section{B. Signal Modeling}

When the antenna is pointed toward a source such as a distant spacecraft, the "residual carrier" portion of the signal can be filtered out and used to estimate the desired parameters as shown in [1]. The signal in the $k$-th channel (out of seven) can be represented as

$$
r_{k}(t)=s_{k}(t)+n_{k}(t) \quad k=1,2,3, \ldots, 7
$$

where the real signal and background noise components are defined as

$$
\begin{aligned}
s_{k}(t) & =\sqrt{2} S_{k} \cos \left(\omega t+\theta_{k}\right) \\
n_{k}(t) & =\sqrt{2}\left[n_{\mathrm{ck}}(t) \cos (\omega t)-n_{\mathrm{sk}}(t) \sin (\omega t)\right]
\end{aligned}
$$

where $n_{\mathrm{ck}}(t)$ and $n_{\mathrm{sk}}(t)$ are uncorrelated baseband random processes representing the in-phase and quadrature components of the noise, and $\omega$ represents the Ka-band $(32 \mathrm{GHz}$ ) carrier frequency [4]. Both the amplitude $S_{k}$ and phase $\theta_{k}$ of the signal depend on the distortion of the antenna, and also on the pointing offset.

Each channel is downconverted to an intermediate frequency of $300 \mathrm{MHz}$, after which spacecraft frequency predicts ${ }^{1}$ derived from the known spacecraft trajectory are applied and the signals are downconverted to baseband. The resulting complex baseband signals are sampled and "frequency-locked" using a digital frequency-lock loop which eliminates any remaining frequency offsets.

The complex samples so obtained remain essentially constant over time-scales of minutes, depending on the antenna dynamics, and contain all of the amplitude and phase information used by the tracking and combining algorithms [3]. During actual tracking, sampling rates of 128 samples per second are typical. These samples can be represented as

$$
\tilde{r}_{k}(i)=\tilde{s}_{k}(i)+\tilde{n}_{k}(i) \quad k=1,2,3, \ldots, 7 \quad i \in Z
$$

where

$$
\tilde{s}_{k}(i) \equiv S_{k} e^{j \theta_{k}}
$$

with

$$
E\left[\tilde{n}_{k}(i)\right]=0 \quad \text { and } \quad E\left[\left\|\tilde{n}_{k}(i)\right\|^{2}\right]=\operatorname{var}\left[\tilde{r}_{k}(i)\right]=\sigma^{2} \text {. }
$$

We assume that noise samples from different channels are independent, as are different noise samples in the same channel, and the amplitude and phase of the signal are assumed constant over the sampling intervals. It is convenient to represent the received samples as seven-dimensional complex vectors of the form $\tilde{\mathbf{r}}(i)=\tilde{\mathbf{s}}(i)+\tilde{\mathbf{n}}(i)$, where $\tilde{\mathbf{r}}(i)=\left(\tilde{r}_{1}(i), \tilde{r}_{2}(i), \ldots, \tilde{r}_{7}(i)\right)$ and where each component is defined as in (5). In order to reduce the effects of noise, the training data set was formed by averaging the received samples over a large number of consecutive received vectors

$$
\begin{aligned}
\tilde{\mathbf{r}}_{a}(j L) & =\left(\tilde{r}_{a, 1}(j L), \tilde{r}_{a, 2}(j L), \ldots, \tilde{r}_{a, 7}(j L)\right) \\
& =\frac{1}{L} \sum_{i=j L-L+1}^{j L} \tilde{\mathbf{r}}(i) \quad j=1,2,3, \ldots
\end{aligned}
$$

where $\tilde{\mathbf{r}}_{a}(j L)$ is the complex averaged vector at time $j L$, with $\tilde{r}_{a, k}$ referring to its $k$ th complex component; $\operatorname{var}\left(\tilde{\mathbf{r}}_{a, k}(j L)\right)=$ $\sigma^{2} / L$, and $\sigma^{2}$ is the variance of the additive white Gaussian noise samples. Thus, with $L=128$, one-second updates are generated, whereas with $L=1280$ the effective integration time is $10 \mathrm{~s}$. Note that as pointed out above signal amplitudes $S_{k}$ and phases $\theta_{k}$ are assumed to remain constant over these observations.

\section{Problem Statement}

The instantaneous pointing error vector of the antenna can be represented as $(X E L, E L)$, a two-dimensional error vector, where $X E L$ is the incremental pointing error in cross-elevation,

${ }^{1}$ These are predictions of the received spacecraft carrier frequency during the tracking period. The relative motion of the spacecraft, along with Earth's rotation, cause the observed carrier frequency to change as a function of time, making accurate prediction necessary for acquiring and tracking the signal. 
and $E L$ is the incremental pointing error in elevation measured in millidegrees (mdeg).

We seek to compute the mapping from the seven-dimensional averaged voltage vector $\tilde{\mathbf{r}}_{a}$ to the two-dimensional error vector $(X E L, E L)$, as represented by

$$
f\left(\tilde{\mathrm{r}}_{a}\right) \rightarrow\left[\begin{array}{c}
X E L \\
E L
\end{array}\right] .
$$

Residual errors in the voltage vector $\tilde{\mathbf{r}}_{a}$ due to noise cause errors in the estimate of (XEL, EL) even if $f\left(\tilde{\mathrm{r}}_{a}\right)$ is known exactly. However, $f\left(\tilde{\mathbf{r}}_{a}\right)$ is also affected by the physical structure of the antenna, which is not always precisely known and which changes as the antenna ages or is buffeted by wind. The noisy and time-varying nature of $f\left(\tilde{\mathrm{r}}_{a}\right)$ poses an additional challenge.

Previous work has demonstrated successful application of interpolated least squares and radial basis function (RBF) networks to correction of antenna pointing errors, based on data from the AFCS, and with the aim of achieving maximum combined SNR [5], [6].

Two distinct problems will be considered here: acquisition and tracking. The acquisition problem involves the estimation of antenna-pointing offsets over a wide range. For instance, if the antenna's pointing has drifted by $4 \mathrm{mdeg}$ in $X E L$ and $-3 \mathrm{mdeg}$ in $E L$, an acquisition algorithm must be able to estimate the offset vector $(4,-3)$ accurately in order to repoint the antenna in the correct direction. Otherwise, a severe loss of signal power would result due to the 5 mdeg pointing error magnitude on a $70-\mathrm{m}$ antenna, which typically has an 8 mdeg beamwidth at $45^{\circ}$ elevation.

The second problem, tracking, focuses on significantly smaller offsets. After the antenna has been correctly pointed on source by the acquisition algorithm, it remains necessary to keep it pointed on source to within one mdeg total pointing error despite slow drift in antenna pointing. Accordingly, the tracking algorithm must estimate small pointing errors near the center of the $(X E L, E L)$ space accurately, and correct them in real time.

\section{TECHNICAL APPROACH}

Two approaches, one involving a radial basis function network and the other a quadratic interpolated least squares algorithm, were developed to synthesize the function $f\left(\tilde{\mathbf{r}}_{a}\right)$ described by (8). Descriptions of both are given below, followed by results and analysis from extensive experiments on real-world and simulated data.

\section{A. Radial Basis Function Networks}

1) Description: A RBF network was developed and used to estimate antenna pointing errors [7], [8]. The complex voltage of the center horn was always normalized to $1+j 0$, making it

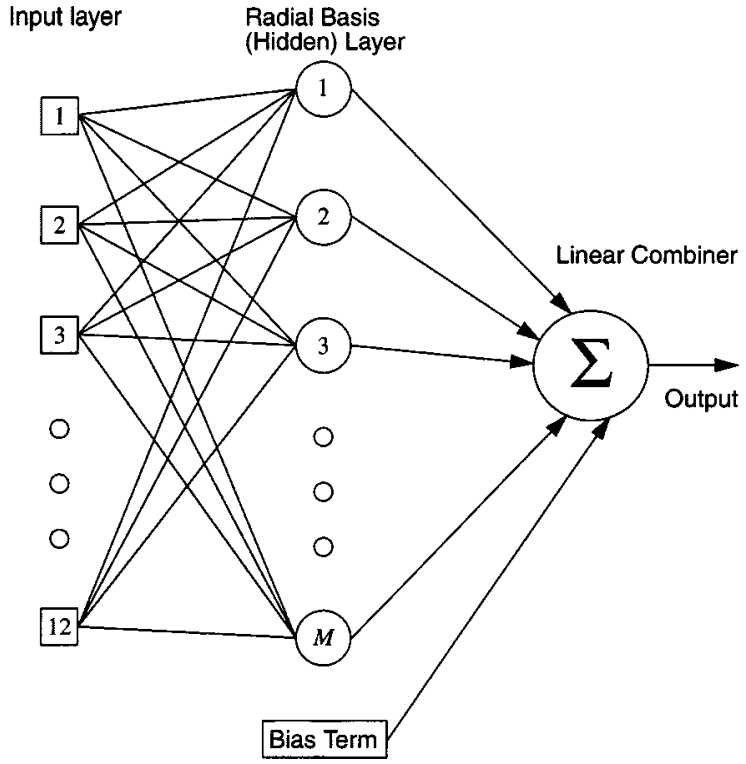

Fig. 2. RBF neural network diagram showing the input layer, the hidden (radial basis) layer, and the output layer (linear combiner) along with the bias term.

unnecessary to provide this input to the network. This normalization eliminates some of the possible time-dependent variations in the received signal, at the cost of a slight reduction in the total information presented to the algorithms. The network's inputs, therefore, consist of the real and imaginary components of the six normalized horn voltages from the outer horns, for an input vector dimension of 12 as shown in Fig. 2. The network was trained to generate values for the incremental $E L$ and $X E L$ offsets corresponding to these inputs.

Each of the $M$ radial basis units implements a Gaussian function of the form

$$
\begin{aligned}
G\left(\tilde{\mathbf{r}}_{a}(j L) ; \mathbf{c}_{i}\right) & =\exp \left(-\left(b\left\|\tilde{\mathbf{r}}_{a}(j L)-\mathbf{c}_{i}\right\|\right)^{2}\right) \\
& =\exp \left(-\left(b \sum_{k=1}^{12}\left(\tilde{r}_{a}(j L)_{k}-c_{i k}\right)\right)^{2}\right)
\end{aligned}
$$

where $\tilde{\mathbf{r}}_{a}(j L)$ is the 12-element averaged input voltage vector at time $j L$ [see (7)], $\mathbf{c}_{i}$ denotes the $i$ th radial basis center, and $b=$ $0.8326 / \sigma_{s}$ controls the width of the unit's region of response. The scalar $b$ is defined so that $G\left(\tilde{\mathbf{r}}_{a}(j L) ; \mathbf{c}_{i}\right)=0.5$ when $\sigma_{s}=$ $\left\|\tilde{\mathbf{r}}_{a}(j L)-\mathbf{c}_{i}\right\|$.

Defining the matrix of hidden layer responses, in Fig. 2, to $N$ consecutive input vectors as shown in (10) at the bottom of the page and defining the linear combiner weight matrix as

$$
\mathbf{w}=\left[\begin{array}{lllll}
w_{1,1} & w_{2,1} & \ldots & w_{M, 1} & c_{\text {bias }, 1} \\
w_{1,2} & w_{2,2} & \cdots & w_{M, 2} & c_{\text {bias }, 2}
\end{array}\right]^{T}
$$

$$
\mathbf{G}=\left[\begin{array}{ccccc}
G\left(\tilde{\mathbf{r}}_{a}(L) ; \mathbf{c}_{1}\right) & G\left(\tilde{\mathbf{r}}_{a}(L) ; \mathbf{c}_{2}\right) & \ldots & G\left(\tilde{\mathbf{r}}_{a}(L) ; \mathbf{c}_{M}\right) & 1 \\
G\left(\tilde{\mathbf{r}}_{a}(2 L) ; \mathbf{c}_{1}\right) & G\left(\tilde{\mathbf{r}}_{a}(2 L) ; \mathbf{c}_{2}\right) & \ldots & G\left(\tilde{\mathbf{r}}_{a}(2 L) ; \mathbf{c}_{M}\right) & 1 \\
\vdots & \vdots & \ddots & \vdots & \vdots \\
G\left(\tilde{\mathbf{r}}_{a}(N L) ; \mathbf{c}_{1}\right) & G\left(\tilde{\mathbf{r}}_{a}(N L) ; \mathbf{c}_{2}\right) & \ldots & G\left(\tilde{\mathbf{r}}_{a}(N L) ; \mathbf{c}_{M}\right) & 1
\end{array}\right]
$$


the output of the radial basis network in response to the $N$ input voltage vectors $\tilde{\mathbf{r}}_{a}(L)$ through $\tilde{\mathbf{r}}_{a}(N L)$ becomes

$$
\mathbf{y}=\left[\begin{array}{llll}
y_{1,1}(L) & y_{2,1}(2 L) & \ldots & y_{N, 1}(N L) \\
y_{1,2}(L) & y_{2,2}(2 L) & \ldots & y_{N, 2}(N L)
\end{array}\right]^{T}=\mathbf{G w}
$$

where $y_{j, 1}(j L)$ and $y_{j, 2}(j L)$ are the RBF network's responses (estimates of $E L$ and $X E L$ ) to the $j$ th averaged input vector $\tilde{\mathbf{r}}_{a}(j L)$ defined in (7) for $j=1,2,3, \ldots$.

For acquisition purposes, dual output networks (for $E L$ and $X E L)$ as described above were designed and tested. For the fine tracking case, it was found that best performance could be achieved, with low complexity, using two single-output networks (one for $E L$ and one for $X E L$ ).

Differences in the antenna's distortion at different antenna elevations led to the training of separate RBF networks for gross elevations of 15,45 , and $75^{\circ}$. The selection of radial basis widths was guided by distances among the voltage vectors in the training set and by experimentation. Since different networks were used for $X E L$ and for $E L$ in the fine tracking case, a total of six networks were designed and evaluated for tracking. For the coarse acquisition case, offset estimates were obtained by a single network at each elevation.

The number of basis units varies depending on the complexity of the function being approximated and may be quite large in certain cases. In our case, the number of basis units was determined by the computationally efficient orthogonal least squares (OLS) algorithm described in [8]. This algorithm uses training data points as radial basis function centers, and the weights are obtained as the solution to a least-squares fitting problem. Although it is possible to use larger radial basis spreads $\sigma_{s}$ to cover a wider area in voltage space, the function defined in (8) becomes increasingly complex as we move away from the origin, or "on-source" direction $(0,0)$ in antenna offset space. This rise in complexity in turn makes it difficult to generate good approximations to $f\left(\tilde{\mathbf{r}}_{\mathbf{a}}\right)$ without using a large number of units, even if larger radial basis spreads $\sigma_{s}$ are used. It was found by experimentation that six to 15 basis units were required with spreads $\left(\sigma_{s}\right)$ of 0.625 to 0.750 over an elevation range of 15 to $75^{\circ}$. For acquisition over the same elevation range, the numbers increase to 77 to 127 basis units, with spreads ranging from 0.50 to 2.50 .

2) Adaptation: In the real world, antenna aging, thermal gradients, and other factors lead to changes in the antenna structure that change the mapping from the 12-dimensional voltage space to the two-dimensional $(X E L, E L)$ space. This makes it necessary to continually and incrementally adapt the RBF network mapping to these changes as new data become available.

Gradient descent-based learning can be used to adapt all of the parameters of an RBF network including the radial basis spreads and the radial basis centers themselves. While this procedure can adjust all of the network's parameters over time (except for the number of basis units, which remains fixed), it does not always converge. By contrast, for a fixed radial basis layer, it is possible to achieve a global minimum of the meansquared error as a function of the linear combiner weights, and good retraining performance is significantly easier to achieve. We take this approach here, and describe two algorithms which avoid the need to generate an entirely new radial basis network using the OLS procedure every time the antenna changes, resulting in computational savings and the ability to adjust the RBF network in near real time.

3) Least mean squares (LMS) and Pseudoinverse Algorithms: Since the linear combiner in the RBF network may be thought of as a linear adaptive filter, the LMS algorithm may be applied to adjust its weights and bias term. The linear combiner calculates

$$
y(n)=c_{\mathrm{bias}}(n)+\sum_{i=1}^{N} w_{i}(n) G\left(\mathbf{x}(n) ; \mathbf{c}_{i}\right) .
$$

Updates of the weights are given by

$$
w_{i}(n+1)=w_{i}(n)+\eta e(n) G\left(\mathbf{x}(n) ; \mathbf{c}_{i}\right)
$$

where $e(n)=d(n)-y(n)$ is the error formed between the desired output $d(n)$ and the actual output $y(n)$. Here $\eta$ is the learning rate parameter, which can be adaptively updated for faster convergence [9]. The bias term is viewed as a weight attached to a fixed input of +1 and updated via

$$
c_{\text {bias }}(n+1)=c_{\text {bias }}(n)+\eta e(n) .
$$

While the LMS algorithm is a powerful procedure, it is a stochastic approximation to true gradient descent. It is possible to compute the set of linear coefficients for a given training set and fixed radial basis layer which yields the lowest possible mean square error (MSE) [7], [10] as follows.

The matrix $\mathbf{G}$ defined by (10) has a pseudoinverse given by

$$
\mathbf{G}^{+}=\left(\mathbf{G}^{T} \mathbf{G}\right)^{-1} \mathbf{G}^{T} \text {. }
$$

The set of linear combining weights that minimizes the MSE over the training data is

$$
\mathbf{w}_{\text {new }}=\mathbf{G}^{+} \mathbf{d}
$$

where $\mathbf{d}$ is the set of desired outputs at times one through $N$ defined by

$$
\mathbf{d}=[d(1), d(2), \cdots, d(N)]^{T} .
$$

Equation (17) always computes the best possible set of weight updates with respect to the mean-squared error criterion over the set of training vectors.

\section{B. Quadratic Interpolated Least Squares}

A second approach for approximating the mapping described in (8) involves the use of the quadratic interpolated least squares algorithm.

Consider two vector spaces: a 12-dimensional voltage space and a two-dimensional $(X E L, E L)$ space. An initial estimate of the antenna pointing offset is obtained by finding the voltage vector in the training (reference) set closest to the observed voltage vector. The corresponding vector in $(X E L, E L)$ space represents our initial estimate of the pointing offset: let us call it $\left(X E L_{\text {initial }}, E L_{\text {initial }}\right)$.

Next, the eight closest points to $\left(X E L_{\text {initial }}, E L_{\text {initial }}\right)$ in the training set are selected. Using the resulting nine points, one can use one of two methods to obtain the pointing estimates 
$\left(X E L_{\mathrm{est}}, E L_{\mathrm{est}}\right)$. The first method uses all eight points and computes a best fit quadratic surface. The coordinates $(X E L$, $E L)$ where the minimum of this surface occurs, are taken to be the best estimates of the pointing offset. However, it was found that this method offers only a limited advantage over a much simpler quadratic interpolation method, where separate one-dimensional quadratic "slices" are used for XEL and for $E L$ instead of the more complex two-dimensional surface: this simpler method was used in the simulations.

\section{SIMULATION DESCRIPTION}

\section{A. Simulation of the Antenna With Gravitational Distortion}

Spacecraft signals received by the seven-channel AFCS were simulated in two steps. First, a physical optics analysis code was used to obtain the electromagnetic field at the focal plane of the antenna where the apertures of the feed horns are located. This code used the 70-m antenna surface-distortion data obtained from holography measurements during the recent holography-cone experiments [3]. The surface distortions were actually measured at $12.7,37$, and $47^{\circ}$ elevation and were subsequently interpolated to obtain distortions at other elevations. The resulting distortions were added to the nominal surface data to create the final "distorted" reflector surface data. The field at the feed horns in the focal plane was computed by applying a plane wave incident on the main reflector surface, and by tracing the fields to the focal plane via the subreflector.

Next, the computed field data were used to obtain the complex voltages at the horn array, and the field and power over the aperture of each individual horn was determined. The difference between the power captured by the feed horns and the total power at the focal plane is an indication of feed array efficiency. In a separate calculation, the fields at the aperture of each horn, induced by the application of a unit voltage to the input of the horn, were calculated using a theoretical waveguide modal expansion. These fields were subsequently integrated over each horn in the focal plane in order to calculate the final complex voltages.

Figs. $3-5$ show the normalized received power distribution on the focal plane of the reflector at $15^{\circ}, 45^{\circ}$, and $75^{\circ}$ elevations, respectively. The feed apertures of the array are superimposed on the plots to indicate the power distribution captured by each horn. It can be seen that at $45^{\circ}$, where the reflector surface is designed to provide "optimal" performance, most of the energy is captured by the center horn. On the other hand, at $15^{\circ}$ and $75^{\circ}$ elevations, where the effects of gravitational distortions are significant, considerable energy falls beyond the center horn but is largely recovered by the ring of array elements. As can be seen, the effects of distortions are particularly pronounced at $75^{\circ}$ elevation angle. Fig. 5 shows that even in the presence of the surrounding feed elements some of the energy falls between the horns and therefore is not fully recovered. However, simulations also show that the surrounding ring elements can be rotated to a position that increases the captured signal power by more than $0.5 \mathrm{~dB}$. Furthermore, an additional $1 \mathrm{~dB}$ in efficiency can be obtained by optimally repositioning the entire array in the focal plane, or equivalently repointing the antenna in the direction that yields maximum combined SNR.

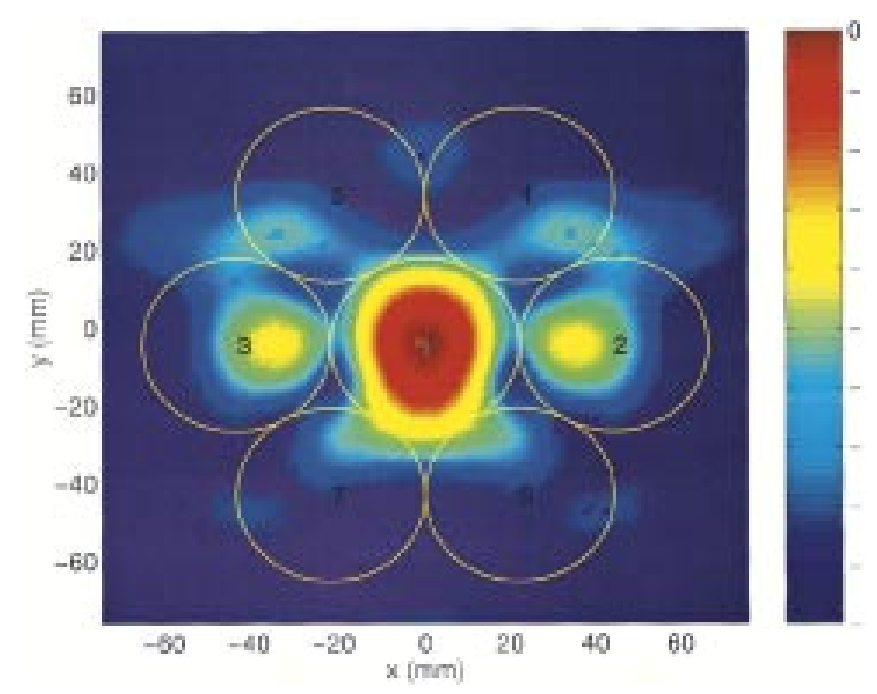

Fig. 3. Normalized received power distribution (dB) on focal plane of 70-m reflector. Elevation: $15^{\circ}$.



Fig. 4. Normalized received power distribution (dB) on focal plane of $70-\mathrm{m}$ reflector. Elevation: $45^{\circ}$.

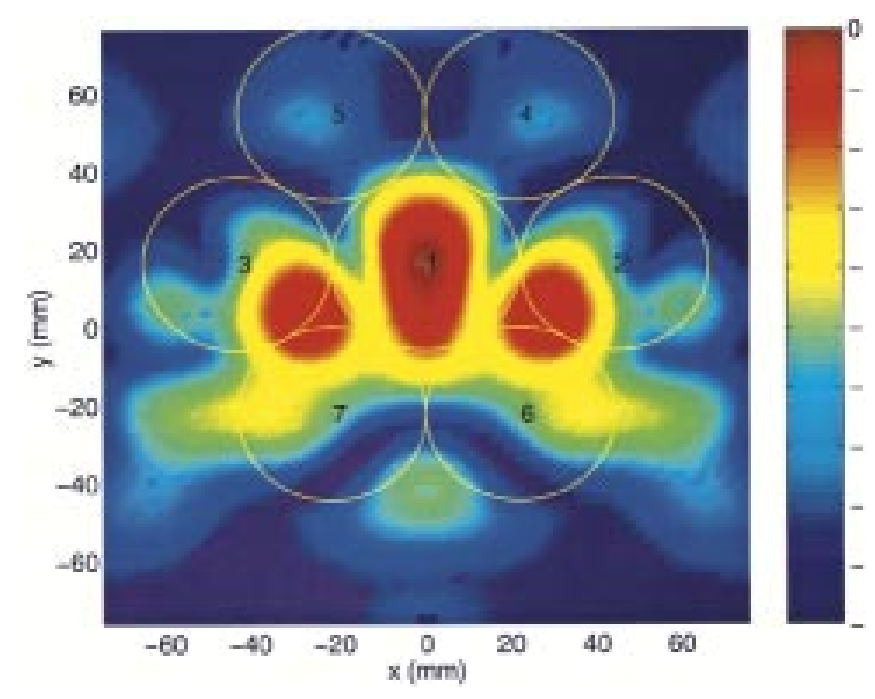

Fig. 5. Normalized received power distribution (dB) on focal plane of 70-m reflector. Elevation: $75^{\circ}$. 
TABLE I

Test Regions for Tracking Performance Evaluation

\begin{tabular}{c||c}
\hline Region 1 & $(0,0)$ \\
\hline Region 2 & Square. $\pm 0.50 \mathrm{mdeg}$ in $X E L$ and in $E L$. \\
\hline Region 3 & Square. $\pm 1.00 \mathrm{mdeg}$ in $X E L$ and in $E L$. \\
\hline Region 4 & Square. $\pm 1.50 \mathrm{mdeg}$ in $X E L$ and in $E L$. \\
\hline Region 5 & Square. $\pm 2.00 \mathrm{mdeg}$ in $X E L$ and in $E L$. \\
\hline
\end{tabular}

\section{B. Tracking Regions}

Although the test sets for tracking were defined over a finely spaced grid ranging from -2.00 mdeg to +2.00 mdeg in both $X E L$ and $E L$, it is still useful to evaluate the algorithms' performance over varying ranges in $(X E L, E L)$ space since, for example, one algorithm may yield better performance very close to the "on-source" direction $(0,0)$ while another may yield better performance over a greater range of pointing offsets. The tracking regions for the simulations are defined in Table I. Error statistics were generated for each of the five tracking regions to illustrate possible performance differences between the RBF network and the quadratic interpolated least squares algorithm.

\section{Simulating Changes in the Antenna Structure}

Both acquisition and tracking performance can be adversely affected by changes in the antenna structure, in which case it becomes necessary for the radial basis networks to adapt to such changes. This is particularly important for fine tracking algorithms since they are responsible for keeping the antenna pointed accurately on source throughout the track.

Changes in the antenna structure were simulated by altering the mapping from voltage space to $(X E L, E L)$ space when generating the training sets for the RBF networks. Let the mapping from voltage space to offset space be given by (8), and create a distorted mapping by changing the $(X E L, E L)$ values corresponding to the voltage vectors using, for example, the following equations:

$$
\begin{aligned}
X E L_{\text {new }} & =\frac{4}{3} X E L_{\text {old }}-\frac{2}{3} \mathrm{mdeg} \\
E L_{\text {new }} & =\frac{4}{5} E L_{\text {old }}-\frac{2}{5} \mathrm{mdeg} .
\end{aligned}
$$

One may think of $X E L_{\text {old }}$ and $E L_{\text {old }}$ as being the correct offsets corresponding to given voltage vectors for the antenna at time $t_{1}$. Antenna aging and other factors may cause the same voltage vectors to map to $X E L_{\text {new }}$ and $E L_{\text {new }}$ at time $t_{2}$, where we have assumed $t_{1}$ and $t_{2}$ are far apart since the idea is to model long-term changes. The RBF networks were trained on the old data in an effort to generate a set of distorted mappings. We can say that these training data were correct at time $t_{1}$ in the distant past but changes in the antenna have yielded a new mapping at time $t_{2}$. We seek to update the RBF network parameters so the networks will accurately determine offsets under the new mapping according to the adaptation strategies discussed in Section II-A2.

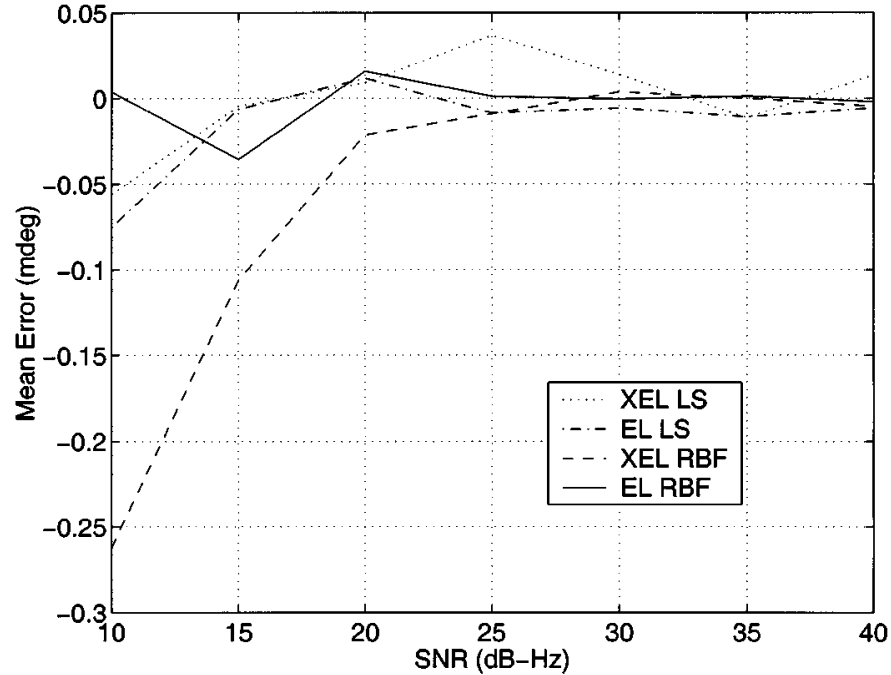

Fig. 6. Acquisition mean errors as a function of SNR for both RBF network and interpolated least squares algorithms at $45^{\circ}$ elevation with 10 -s integration.

\section{Simulation Results}

\section{A. Acquisition Performance Comparison}

One can compute an instantaneous estimate of pointing offset using a single voltage vector sample. This technique corresponds to 1-s averaging, assuming the system is updated at a rate of one sample per second. We can also compute 10 -s averages in which the input voltage vector is averaged over a period of $10 \mathrm{~s}$. The 10-s averaging scheme results in averaged samples which exhibit a factor of ten lower variance than unaveraged 1-s samples. Both techniques were tested for acquisition purposes.

For radial basis networks, mean errors with 10-s averaging were found to be typically less than $0.1 \mathrm{mdeg}$ for SNR above $20 \mathrm{~dB}-\mathrm{Hz}$, as illustrated by Fig. 6, which is representative of results achieved at other elevations. It should be noted that a 10-dB increase in SNR is associated with 10-second integration, resulting in smaller mean errors.

Fig. 7 illustrates the error standard deviation of both the RBF network and the quadratic least-squares algorithm with 10-s integration. In this figure, the error standard deviations have been averaged over the entire "acquisition range." The error in XEL and the error in $E L$ were calculated for each of these grid points. The root mean square (rms) error in each variable (in $X E L$ and in $E L$ ) was then computed over the entire acquisition test grid. The results shown in this figure are representative of those achieved at other elevations. At high SNR the quadratic least squares algorithm's performance does not improve with increasing SNR as expected, but tends to approach an rms "error floor" of a few tenths of a mdeg. This irreducible error appears to be due to decreased accuracy of the interpolation algorithms near the outer edges of the acquisition range, where simple approximations to the error surface tend to break down. For medium to high SNR (greater than $20 \mathrm{~dB}-\mathrm{Hz}$ ), the radial basis network outperforms the least squares algorithm in acquisition mode, whereas in the low SNR region from $10 \mathrm{~dB}-\mathrm{Hz}$ to approximately $15 \mathrm{~dB}-\mathrm{Hz}$ the least squares algorithm yields best performance. These results suggest the use of a hybrid system consisting of a least squares 


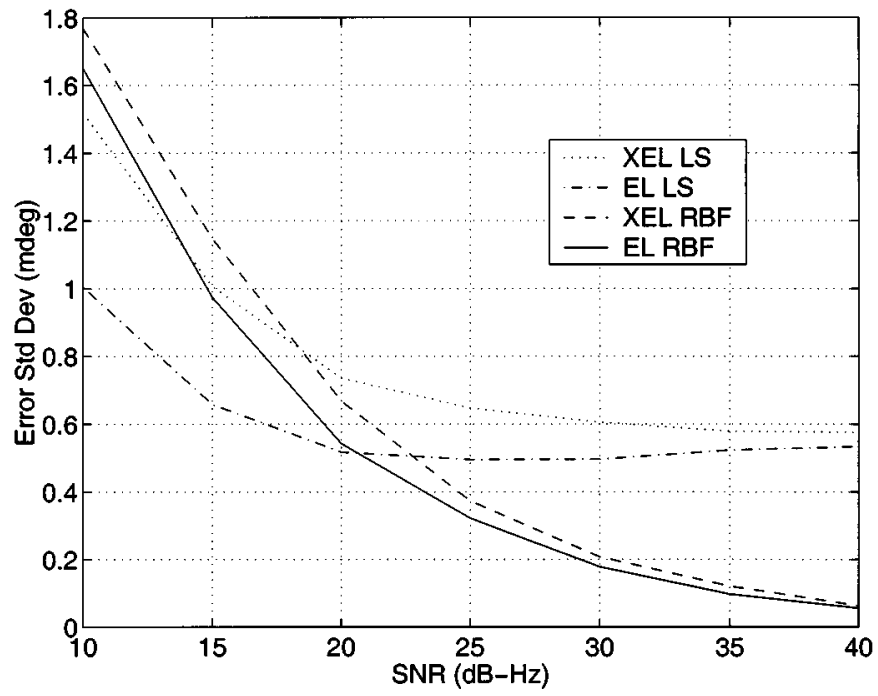

Fig. 7. Acquisition error standard deviations as a function of SNR for both RBF network and interpolated least squares algorithms at $45^{\circ}$ elevation with 10 -second integration.

algorithm for low SNR acquisition, and an RBF network for the medium to high SNR regions.

In summary, these simulations have demonstrated that the RBF network yields better mean error and rms error performance than interpolated least squares in the medium to high SNR range during acquisition. However, under very noisy conditions in acquisition mode the quadratic interpolated least squares algorithm yields the lowest rms and mean errors.

\section{B. Tracking Performance Comparison}

Tracking involves estimation and correction of small pointing errors. Tracking algorithms need only be optimized for accurate estimation of errors over a small range: our tests were performed by applying offsets ranging from $-2.00 \mathrm{mdeg}$ to $2.00 \mathrm{mdeg}$ in both $X E L$ and $E L$.

As previously discussed, five tracking regions were defined for evaluation purposes. The first region was really the point $(0$, $0)$ in $(X E L, E L)$ space, where the mean error and error standard deviations of the estimates were determined with the simulated antenna pointing perfectly on source in the presence of noise. The next region is a square centered at $(0,0)$, and the remaining regions are progressively larger squares, as defined in Table I.

1) RBF Network Performance: Figs. 8 and 9 show the desired target points in $(X E L, E L)$ space denoted by dark " $\times$ " symbols and the estimates computed by the RBF networks as light dots. For each offset, shown as a dark " $\times$, , 100 independent estimates from the RBF network were used to obtain a scatter plot of the networks' estimates of the applied antenna offsets. These light dots form clusters near the target points, and the size and the center of each cluster gives a rough indication of network performance.

Each scatter plot was taken at an SNR of $40 \mathrm{~dB}-\mathrm{Hz}$ with 10 -second integration, illustrating performance over region 3 as defined in Table I. It is interesting to observe that performance is better at higher elevations, where there is significant antenna distortion, than at $15^{\circ}$ elevation where distortion is less severe. This is attributed to the fact that more signal power is present in

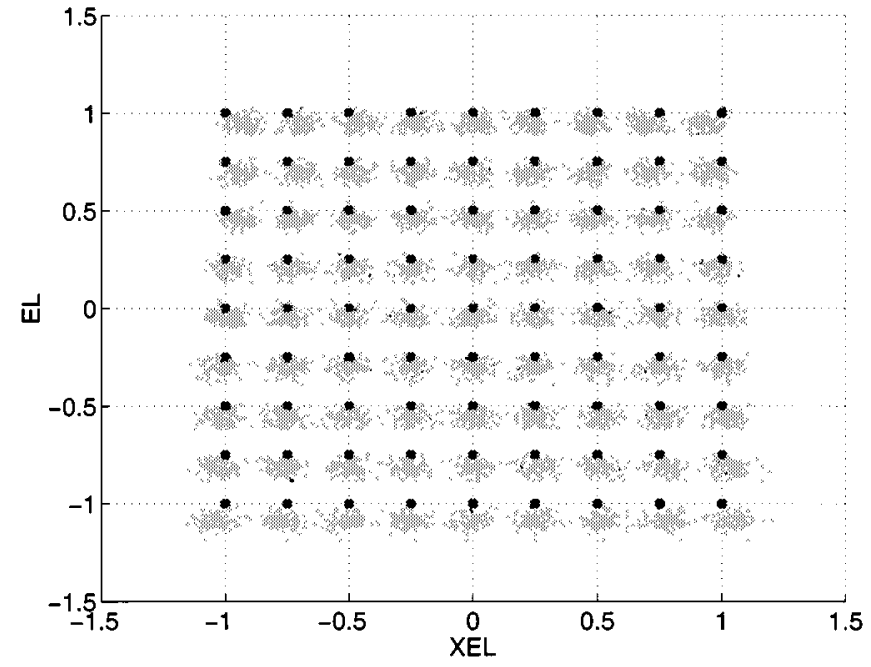

Fig. 8. RBF Region 3: $15^{\circ}, 40 \mathrm{~dB}-\mathrm{Hz}, 10-\mathrm{s}$ integration, Tracking mode. Region 3 is a square region in pointing offset space ranging over $\pm 1 \mathrm{mdeg}$ in both $X E L$ and $E L$. The light gray clouds are RBF network estimates of the true pointing offsets, which are given by the dark " $X$ " symbols.

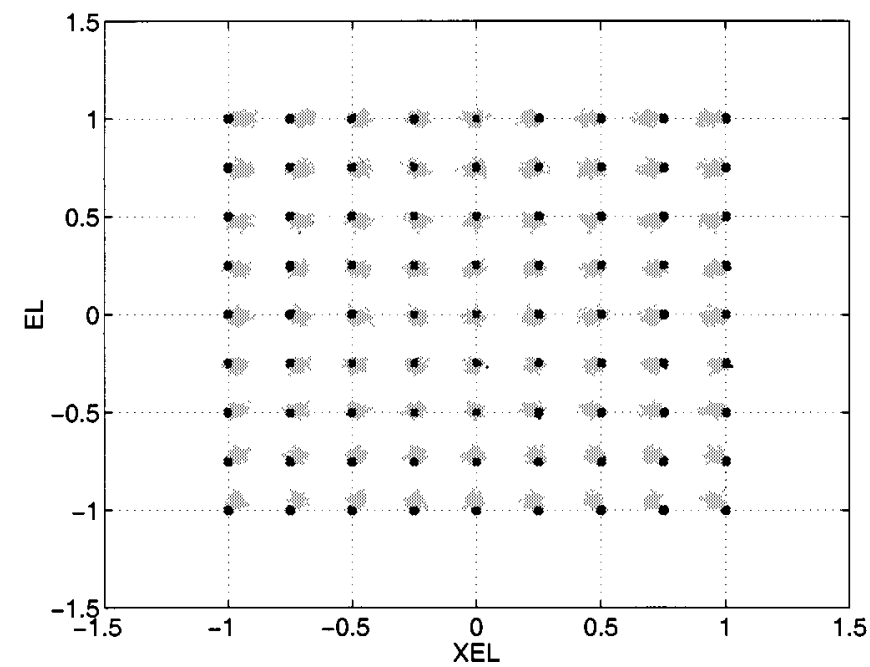

Fig. 9. RBF Region 3: $75^{\circ}, 40 \mathrm{~dB}-\mathrm{Hz}, 10-\mathrm{s}$ integration, Tracking mode Region 3 is a square region in pointing offset space ranging over $\pm 1 \mathrm{mdeg}$ in both $X E L$ and $E L$. The light gray clouds are RBF network estimates of the true pointing offsets, which are given by the dark " $X$ " symbols.

the outer horns when there is distortion, providing more information to the RBF network for a better estimate of the applied offset. However, we also observe a slight bias at $75^{\circ}$ of elevation, evidenced by the fact that the clusters of estimates (light dots) are not centered perfectly on the true offsets (dark " $X$ " symbols). A summary of RBF network performance in terms of mean error and error standard deviation at $40 \mathrm{~dB}-\mathrm{Hz}$ SNR, with 10-s integration, is provided in Table II.

2) Least Squares Performance: A scatter plot for the quadratic interpolated least squares algorithm's estimates is given in Fig. 10. At $75^{\circ}$ elevation estimator bias starts to become significant. The error standard deviation and mean error of the least squares algorithm, averaged over the entire grid, are shown in Table III.

3) Performance Comparison: The least squares algorithm evaluated here implicitly assumes a quadratic error surface since it uses quadratic interpolation. This assumption appears to be 
TABLE II

RBF Network Tracking Performance at $40 \mathrm{~dB}-\mathrm{Hz}$ WITH 10-S INTEGRATION

\begin{tabular}{c|l|c|c}
\hline Elevation (deg) & Variable & Mean Error (mdeg) & Error Standard Deviation (mdeg) \\
\hline \hline 15 & $X E L$ & -0.0005 & 0.0491 \\
\hline 15 & $E L$ & 0.0434 & 0.0350 \\
\hline 45 & $X E L$ & 0.0009 & 0.0696 \\
\hline 45 & $E L$ & 0.0231 & 0.0523 \\
\hline 75 & $X E L$ & 0.0052 & 0.0380 \\
\hline 75 & $E L$ & 0.0090 & 0.0258 \\
\hline
\end{tabular}

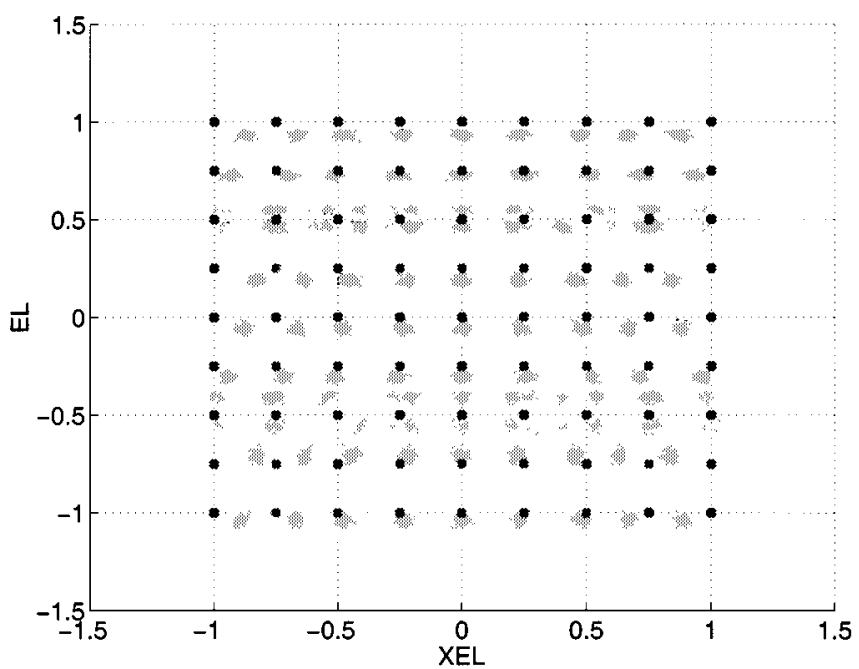

Fig. 10. LS Region 3: $75^{\circ}, 40 \mathrm{~dB}-\mathrm{Hz}, 10$-s integration, Tracking mode. Region 3 is a square region in pointing offset space ranging over $\pm 1 \mathrm{mdeg}$ in both $X E L$ and $E L$. The light gray clouds are RBF network estimates of the true pointing offsets, which are given by the dark " $x$ " symbols.

good at both 15 and $45^{\circ}$ elevation where performance is nearly identical to that of the RBF networks.

At $75^{\circ}$ elevation, the antenna surface suffers significant distortion. The spatial distribution of power in the focal plane is affected by these distortions, and a significant amount of power appears in the outer horns. Furthermore, the assumption of a quadratic error surface only appears to hold for a very small neighborhood in this region, with a breakdown of the quadratic assumption becoming evident as we move away from $(0,0)$ in antenna offset space as shown in Fig. 10.

The RBF network is better able to deal with severe antenna distortion as illustrated in Fig. 9 at $75^{\circ}$ elevation. Under the high SNR conditions shown in Figs. 9 and 10, the clusters are very small for both algorithms at $75^{\circ}$ elevation. The errors in the least squares algorithm at this elevation are mostly systematic with very little random error, indicating breakdown of the quadratic surface assumption at high elevations when the antenna's main reflector is severely distorted.

Figs. 11 and 12, which highlight performance over region 3, defined over \pm 1.00 mdeg in antenna offset space, are representative of results achieved for all five regions. Fig. 11 shows the mean errors obtained with both the interpolated least squares and RBF network algorithms. Both 1-s and 10-s integration times are included in the error plots. Error standard deviations
TABLE III

QuAdRATIC INTERPOLATED LEAST SQUARES TRACKING PERFORMANCE AT $40 \mathrm{~dB}-\mathrm{Hz}$ WITH 10-s INTEGRATION

\begin{tabular}{c|l|c|c}
\hline Elevation (deg) & Variable & Mean Error (mdeg) & Error Standard Deviation (mdeg) \\
\hline \hline 15 & $X E L$ & 0.0008 & 0.0437 \\
\hline 15 & $E L$ & 0.0154 & 0.0330 \\
\hline 45 & $X E L$ & 0.0001 & 0.0685 \\
\hline 45 & $E L$ & 0.0034 & 0.0633 \\
\hline 75 & $X E L$ & 0.0000 & 0.0819 \\
\hline 75 & $E L$ & 0.0268 & 0.0422 \\
\hline
\end{tabular}

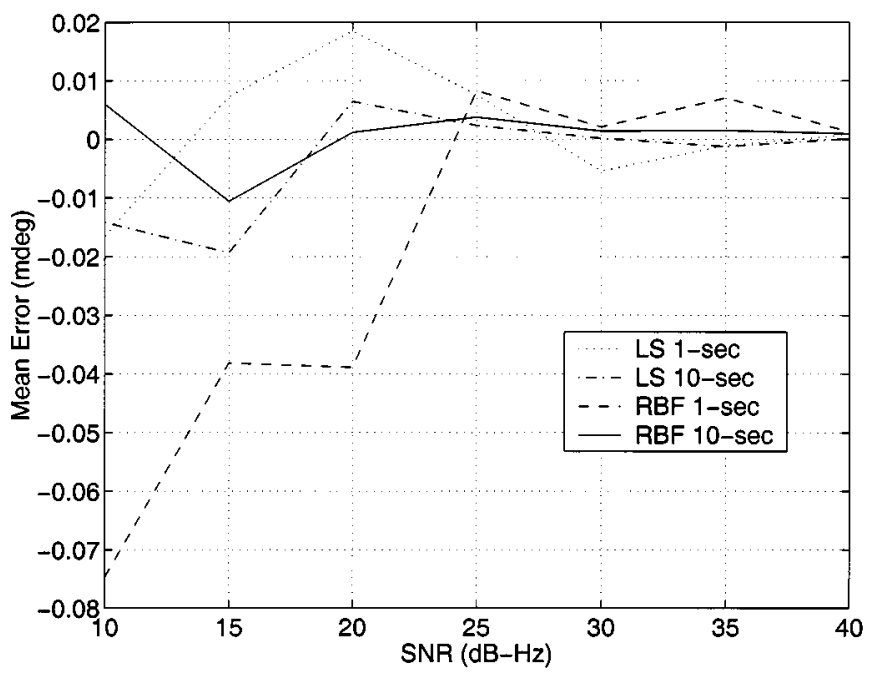

Fig. 11. Tracking mean errors $X E L 45^{\circ}$. We see that the mean estimation error is typically less than 0.1 mdeg even under noisy conditions. This suggests that typical systematic errors in estimation of pointing offsets are fairly small.

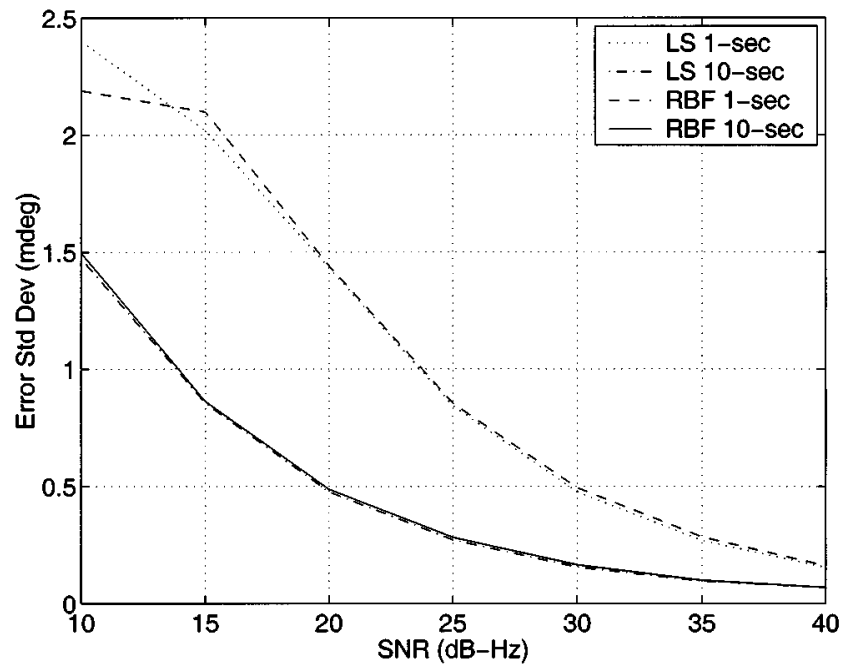

Fig. 12. Tracking error standard deviations $X E L 45^{\circ}$. The error standard deviation is plotted as a function of SNR in $(\mathrm{dB}-\mathrm{Hz})$. Errors in estimating antenna pointing are seen to be largely random with only a negligible systematic component which was illustrated in Fig. 11. We see that the RBF network's performance is very similar to that of the interpolated least squares algorithm used for comparison.

are illustrated in Fig. 12. In all cases, errors in mdeg are plotted against SNR in $\mathrm{dB}-\mathrm{Hz}$. 




Fig. 13. Performance of RBF network trained on old data at $75^{\circ}$. The black " $X$ " symbols denote the true pointing offsets, while the gray patches denote multiple RBF network estimates. Training the RBF network on old data results in significant systematic pointing errors which must be corrected by retraining.

RBF networks generally exhibit higher mean errors at low SNR than the least squares algorithm. At medium-to-high SNR the RBF network's performance does not differ significantly in terms of mean error. Furthermore, the error standard deviations are comparable, indicating the RBF network comes close to the performance of the quadratic interpolated least squares algorithm. For $75^{\circ}$ of elevation, the RBF network exhibits an advantage in terms of overall performance, mostly due to its ability to better handle nonquadratic error surfaces which are characteristic of severely distorted antennas. Overall, both algorithms exhibit similar performance for tracking at elevations of 15 and $45^{\circ}$, while the RBF network performs better than the quadratic interpolated least squares algorithm at very high elevations corresponding to severe antenna distortion.

We observe that both algorithms achieve the nominal 0.8 mdeg total pointing accuracy requirement at SNR above approximately $20 \mathrm{~dB}-\mathrm{Hz}$ at all elevations when $10-\mathrm{s}$ integration is used. This integration time is compatible with the practical update rate of the antenna, and represents outstanding "weak signal" performance. Above $30 \mathrm{~dB}-\mathrm{Hz}$, with 1-s integration, both algorithms meet the 0.8 mdeg requirement corresponding to $0.1 \mathrm{~dB}$ SNR loss, although pointing corrections generally cannot be applied to the antenna at such a high rate.

\section{Adaptive Tracking Results}

As discussed in Section III-C, a simple affine transform was used to simulate a changed antenna and to determine the performance of the radial basis network on translational, shrink, and stretch distortions. Six RBF networks were trained on voltage-tooffset mappings representing the antenna in the "old" state. The affine transform was used to change the voltage-to-offset mapping, and the RBF networks were simulated on the "new" antenna state both before and after undergoing retraining.

In Fig. 13 (40 dB-Hz and 10-s integration times) we see that the estimates (light clusters) corresponding to the antenna offsets from the RBF networks are very far from the dark " $x$ " symbols denoting the new values. This illustrates the performance

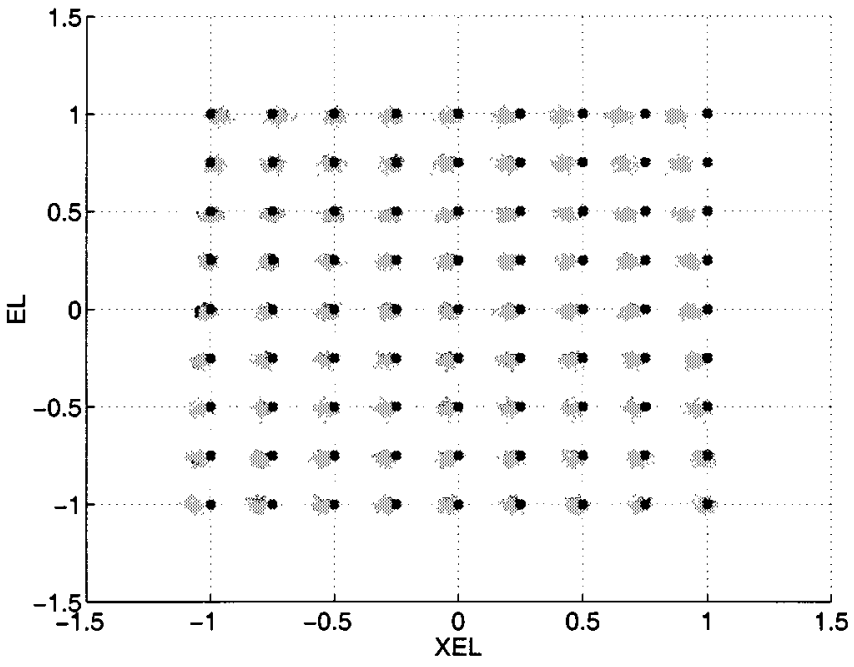

Fig. 14. Performance of RBF network adapted with the LMS algorithm at $75^{\circ}$ The RBF network has been successfully adapted to the new data. Small errors of a fraction of a mdeg remain, as evidenced by the fact that the gray clouds of RBF pointing estimates differ somewhat from the actual pointing estimates given by the black " $x$ " symbols. However, this is a very significant improvement over the performance prior to adaptation illustrated in Fig. 13, and these errors still lie well within the DSN's 0.8 mdeg accuracy requirement.

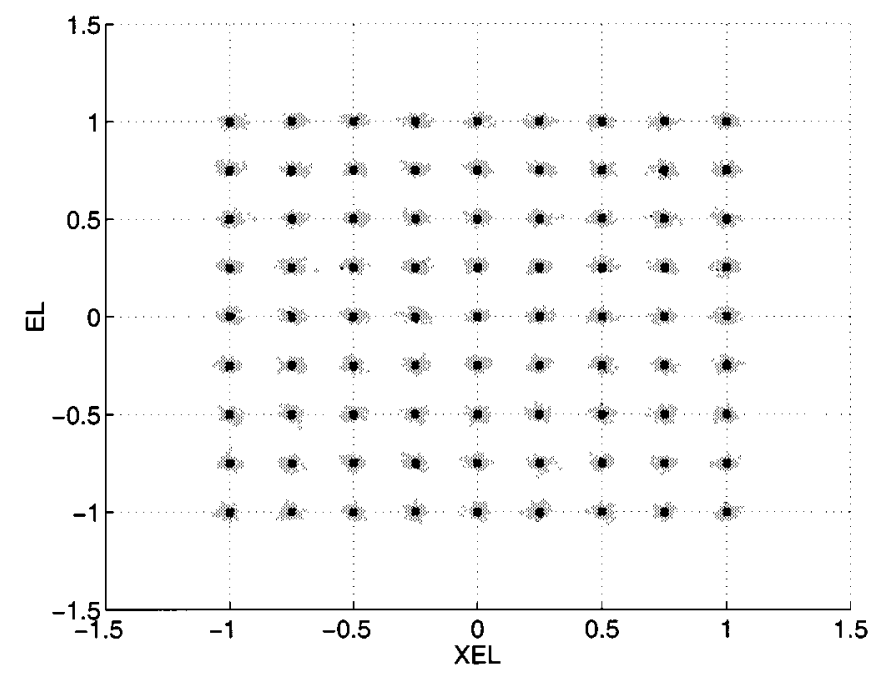

Fig. 15. Performance of RBF network adapted with the pseudoinverse method at $75^{\circ}$. Since the pseudo-inverse method will calculate the best possible linear combiner weights given the new data set and given a fixed radial basis layer, we see that this network performs very well. The clusters of RBF pointing estimates are centered very well on the black " $x$ " symbols denoting actual pointing offsets, illustrating the excellent performance of the pseudo-inverse method in adapting the RBF network.

of the networks trained on old data representing, for example, a pointing bias and scaling due to aging. Our objective is to retrain the networks to remove the pointing bias by adapting their linear combiners.

1) Adaptation Results: Using an updated set of training data, the RBF networks were retrained using the LMS algorithm. The resulting networks, with 10-s integration and an SNR of 40 $\mathrm{dB}-\mathrm{Hz}$, yield the performance illustrated in Fig. 14, which is representative of results achieved at other elevations. Most of the systematic pointing bias has been successfully removed.

The pseudoinverse method yields superior training performance, as shown in Fig. 15, with most of the error caused by the old training set well corrected after retraining is complete. 
TABLE IV

rms ERRORS OBTAINED WHILE TRACKING THE CASSINI SPACECRAFT: No LINEAR TREND SUBTRACTED

\begin{tabular}{c|c|c|c|c|c|c|c}
\hline $\begin{array}{c}\text { Day of } \\
\text { the year }\end{array}$ & $\begin{array}{c}\text { SNR } \\
\text { (dB) }\end{array}$ & $\begin{array}{c}\text { Elevation } \\
\text { (deg) }\end{array}$ & $\begin{array}{c}\text { (A)scend/ } \\
\text { (D)escend }\end{array}$ & RBF/LS & $\begin{array}{c}\sigma_{X E L} \\
\text { (mdeg) }\end{array}$ & $\begin{array}{c}\sigma_{E L} \\
\text { (mdeg) }\end{array}$ & $\begin{array}{c}\sigma_{\text {total }} \\
\text { (mdeg) }\end{array}$ \\
\hline \hline 68 & 44.4 & 62.7 to 59.2 & D & RBF & 0.2274 & 0.7778 & 0.8104 \\
\hline 195 & 45.2 & 39.5 to 41.6 & A & RBF & 0.2454 & 0.2280 & 0.3349 \\
\hline 107 & 50.1 & 41.1 to 39.2 & D & LS & 0.4220 & 0.5167 & 0.6671 \\
\hline
\end{tabular}

TABLE V

rms ERRORS OBTAINED WHILE TRACKING THE CASSINI SPACECRAFT: LINEAR TREND SUBTRACTED

\begin{tabular}{c|c|c|c|c|c|c|c}
\hline $\begin{array}{c}\text { Day of } \\
\text { the year }\end{array}$ & $\begin{array}{c}\text { SNR } \\
\text { (dB) }\end{array}$ & $\begin{array}{c}\text { Elevation } \\
\text { (deg) }\end{array}$ & $\begin{array}{c}\text { (A)scend/ } \\
\text { (D)escend }\end{array}$ & RBF/LS & $\begin{array}{c}\sigma_{X E L} \\
\text { (mdeg) }\end{array}$ & $\begin{array}{c}\sigma_{E L} \\
\text { (mdeg) }\end{array}$ & $\begin{array}{c}\sigma_{\text {total }} \\
\text { (mdeg) }\end{array}$ \\
\hline \hline 68 & 44.4 & 62.7 to 59.2 & D & RBF & 0.2226 & 0.2270 & 0.3179 \\
\hline 195 & 45.2 & 39.5 to 41.6 & A & RBF & 0.2226 & 0.2270 & 0.3179 \\
\hline 107 & 50.1 & 41.1 to 39.2 & D & LS & 0.3969 & 0.5154 & 0.6505 \\
\hline
\end{tabular}

As with the LMS case, the results obtained at $75^{\circ}$ are representative of results obtained at other elevations. The LMS method also yields acceptable performance, but LMS is a stochastic gradient descent procedure. It is, therefore, only an approximation to the true gradient descent algorithm, and even true gradient descent takes many iterations to converge asymptotically to the optimal weight vector w. By contrast, the pseudo-inverse method calculates a set of weights which yields the lowest possible MSE for a fixed radial basis layer [7], [10].

If near real-time updating is desired, it may be advisable to use the LMS algorithm, although storing and averaging data to form new training sets to update the linear combiner weights using the pseudoinverse method would likely yield better performance.

\section{REAL-WORLD ACQUISITION AND TRACKING}

Data collected during observations of the Cassini spacecraft in 2001 were used to evaluate RBF networks and interpolated least squares algorithms on the 70-meter antenna at Goldstone, CA. Since antenna dish deformation is highly elevation dependent, data were gathered and tracking operations were performed at elevations ranging from $6^{\circ}$, the antenna's lower limit, up to $75^{\circ}$, the highest elevation achieved while tracking Cassini.

While tracking the Ka-band carrier, the antenna pointing was refined by means of a "five-point boresighting" algorithm that introduced \pm 4 mdeg pointing offsets in two orthogonal directions along the line-of-sight. The algorithm then measured the signal power in the central horn (channel 1) at each offset as well as with no offset (nominal on-source direction) and computed a pointing update based on a quadratic fit to the data. After a few iterations, the magnitude of the updates approached zero, and the signal power in the central channel was maximized: this was considered to be the true "on-source" direction. The antenna was allowed to track the source using its own tracking model,

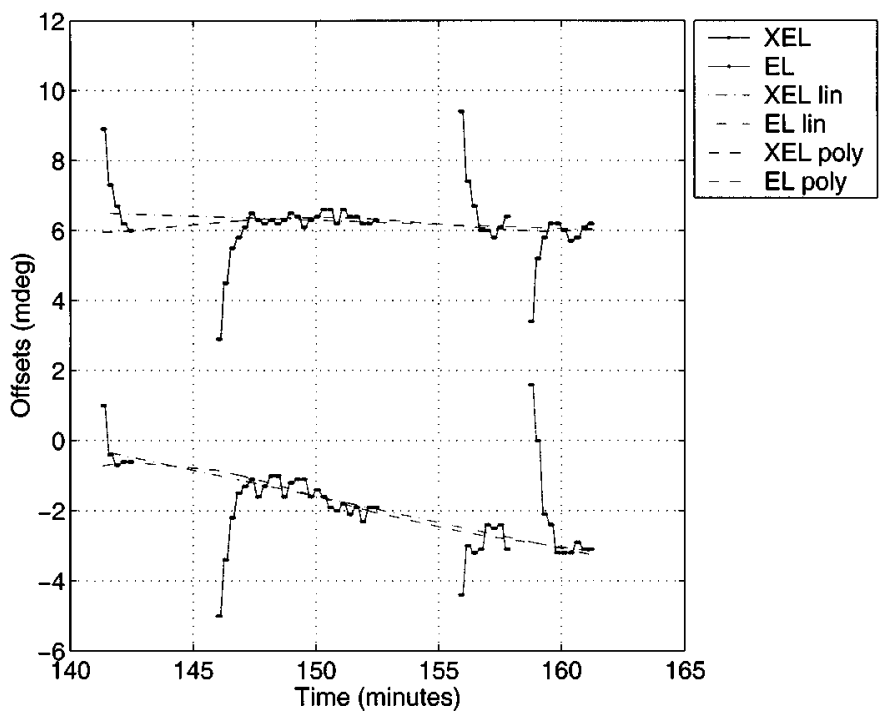

Fig. 16. Day $68 \mathrm{RBF}$ track of Cassini with antenna descending from $62.9^{\circ}$ to $59.2^{\circ}$ elevation. Pointing errors were intentionally applied in order to test the RBF network's ability to recover quickly and accurately. It can be seen that the RBF network quickly returns antenna pointing to the long-term trend line, illustrating the system's ability to keep the antenna pointed accurately on source.

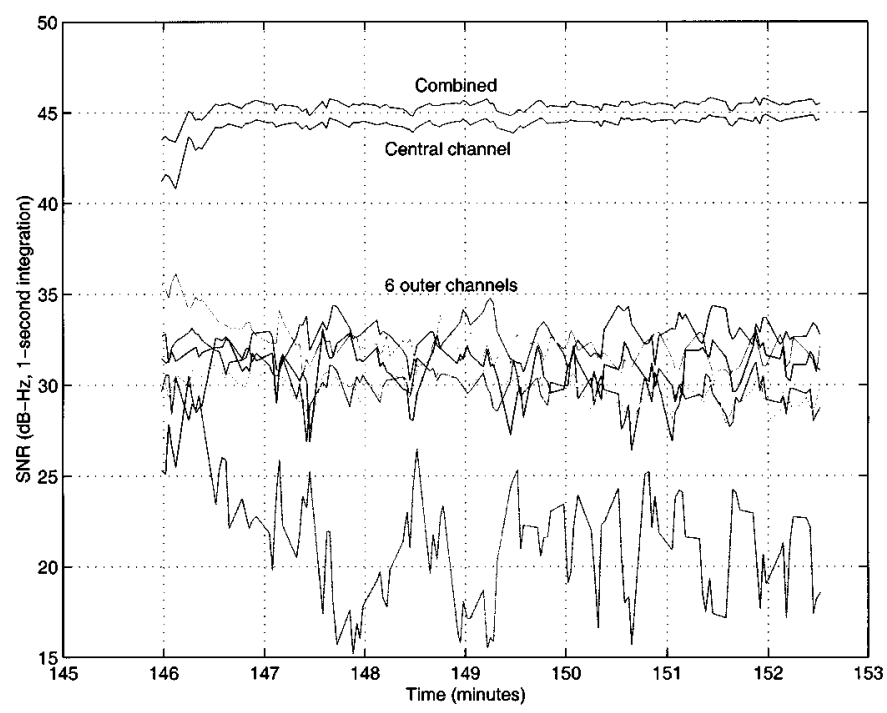

Fig. 17. One of the day $68 \mathrm{RBF}$ tracks of Cassini with antenna descending from $62.9^{\circ}$ to $59.2^{\circ}$ elevation. The RBF network is clearly able to recover from the intentionally applied pointing error, allowing a rapid return to peak SNR operation. The objective here is to show that SNR pointing losses are effectively minimized by the use of the RBF network for pointing control.

which takes into account both earth rotation and relative motion of the spacecraft.

Following this fine-pointing operation, a raster-scan was initiated. The antenna was commanded to various predetermined offsets from the nominal on-source direction, and the received vector measured at each point. The data were taken at offsets ranging from -3.00 to +3.00 mdegs with regular spacing of 1.00 mdeg in both $X E L$ and $E L$ directions. Note that the zerooffset case defines the effect of antenna distortions on the received complex signals at that elevation, whereas the other cases contain a mixture of both distortion and pointing effects. Only the response of the six outer horns were used to train the network, as explained in Section II. 


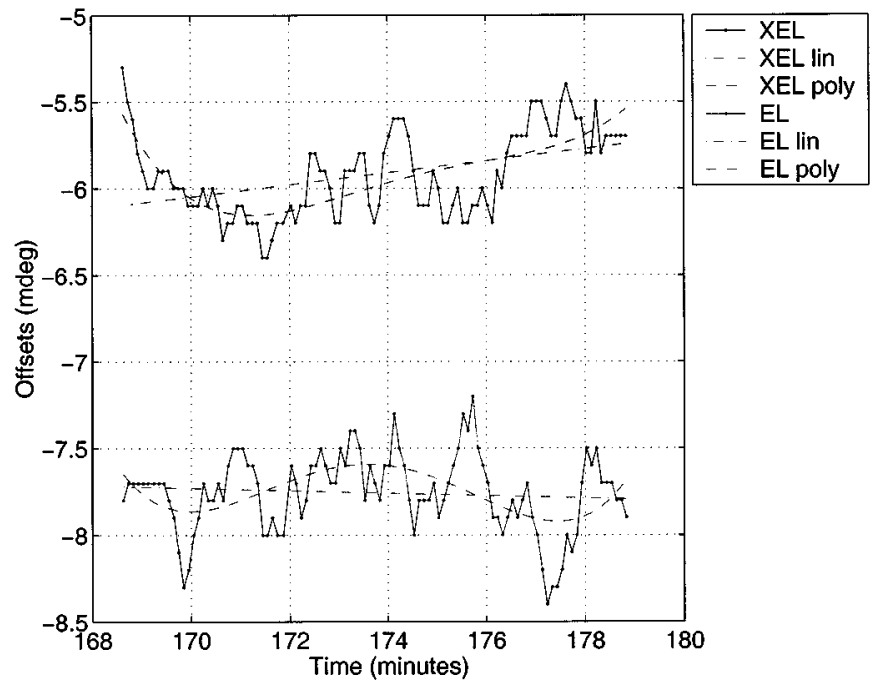

Fig. 18. Day $195 \mathrm{RBF}$ track of Cassini with antenna ascending from 39.5 to $41.6^{\circ}$ elevation. This illustrates the RBF network's steady-state tracking ability. The antenna ran under RBF network control for over $10 \mathrm{~min}$, and the algorithm continued to accurately track the long-term trend in the on-source pointing direction very well.

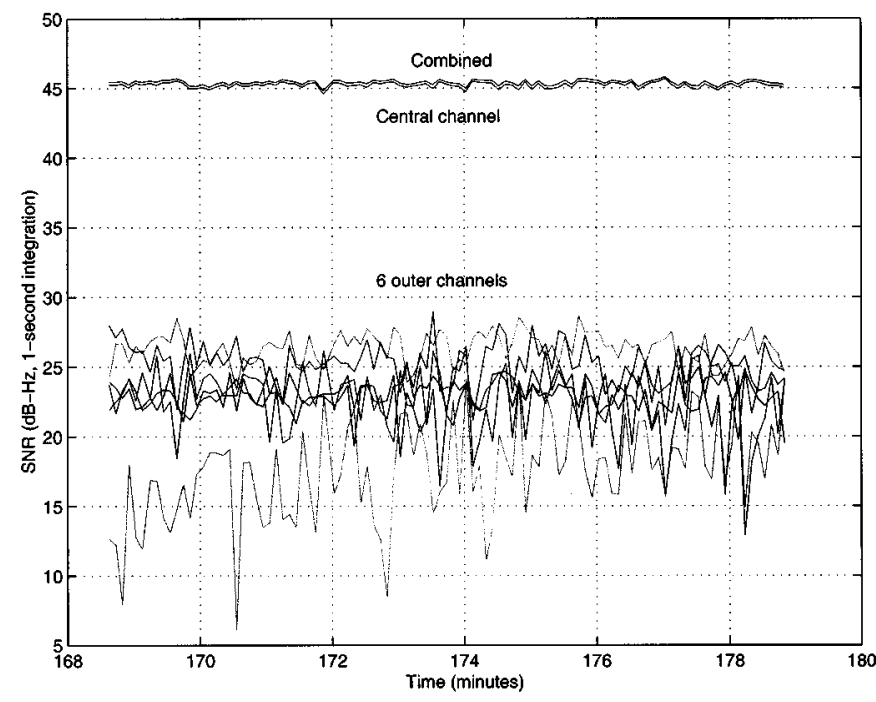

Fig. 19. Day $195 \mathrm{RBF}$ track of Cassini with antenna ascending from 39.5 to $41.6^{\circ} \mathrm{s}$ elevation. The RBF network effectively prevented pointing related SNR losses for a period of over 10 min of steady-state tracking. The stable SNR attests to the overall stability of the algorithm during long tracking periods.

\section{A. Performance Evaluation: Tracking the Cassini Spacecraft}

Cassini tracking operations carried out in 2001 provide a picture of RBF and interpolated least squares capabilities. These operations were carried out in real-time with the algorithms controlling the pointing of the 70-m antenna.

The physical antenna suffers from several possible sources of pointing error which are not modeled in our simulations, including small-scale vibrations of the mechanical structure. However, real-world testing still tends to yield rms pointing errors well under $0.8 \mathrm{mdeg}$, particularly at high SNR. The antenna's gross pointing is controlled by a computerized model which uses "pointing predicts" to determine where the spacecraft should be in the sky. These pointing predicts often have errors in excess of $10 \mathrm{mdeg}$, necessitating the development

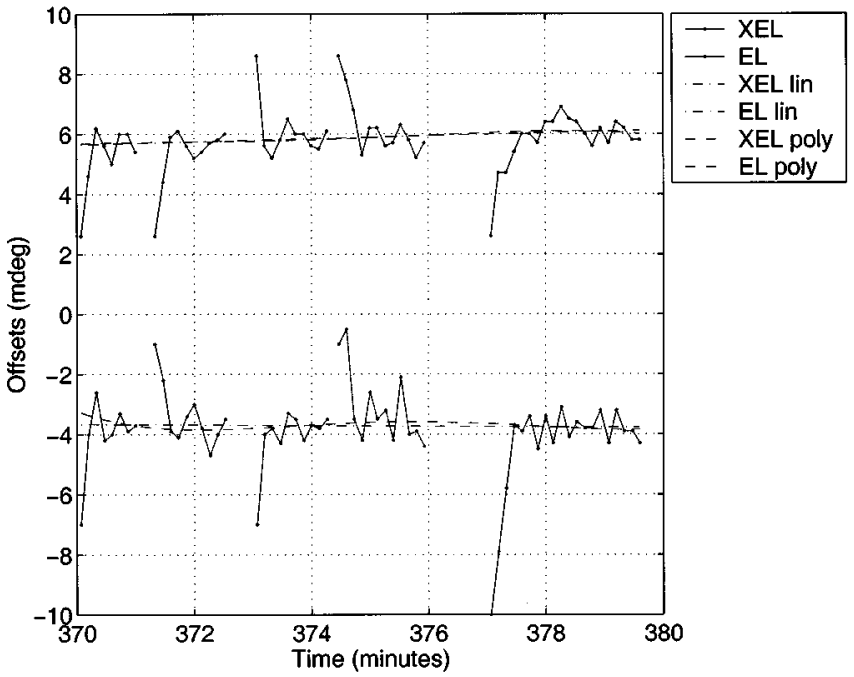

Fig. 20. Day 107 LS track of Cassini with antenna descending from 25.3 to $22.8^{\circ}$ elevation. The interpolated least squares algorithm also does an efficient job of correcting intentionally applied pointing errors, keeping the antenna pointed accurately on-source. Here we see that the intentionally applied offsets were corrected very rapidly, allowing a quick return to the on-source direction.

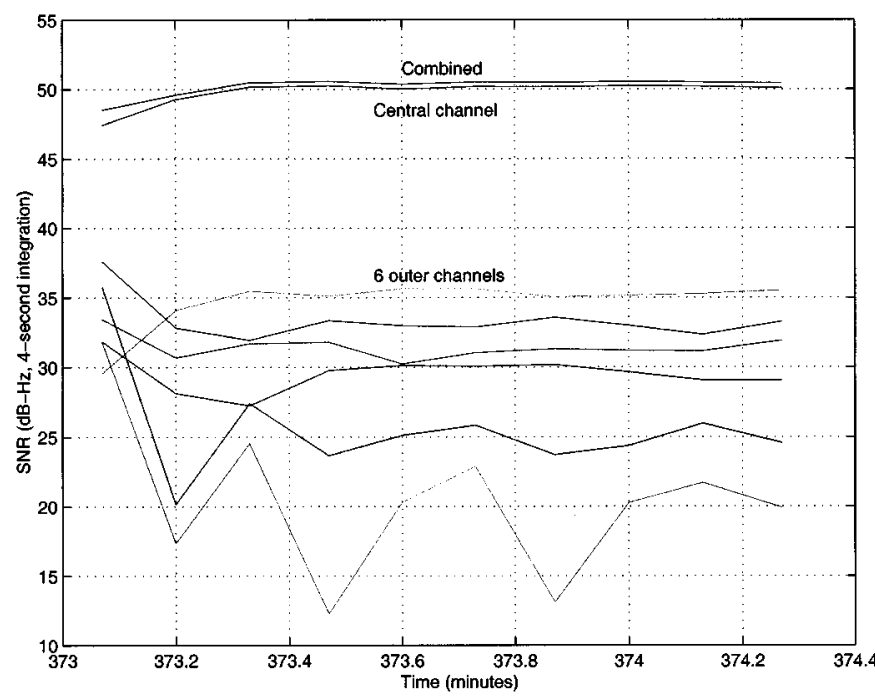

Fig. 21. Expanded version of track \#3 of Fig. 20. A day 107 LS track of Cassini with antenna descending from 25.3 to $22.8^{\circ}$ elevation. SNR recovery is very rapid with the interpolated least squares algorithm, which does an excellent job of correcting pointing-related SNR loss. Here we see the changes in SNR from the time we deliberately apply a pointing error until the algorithm corrects the antenna's pointing, returning SNR to its peak value.

of fine tracking algorithms. For that reason, it is typical for the spacecraft's position in $(X E L, E L)$ coordinate space to drift slowly with respect to the pointing predicts. Any analysis of rms tracking errors must, therefore, involve subtraction of this slow drift from the pointing data. We report here in Tables IV and $\mathrm{V}$ both sample (uncorrected) rms errors, and rms errors with the long-term linear trend removed, for a number of tracks.

On "day 68," that is, 68 days from the beginning of the year 2001, a 1-s integration scheme was used. In this case, five RBF network outputs were averaged in order to calculate each update. Additionally, a delay of several seconds was included to permit the antenna sufficient settling time after each position update command was sent. Consequently, pointing updates were 


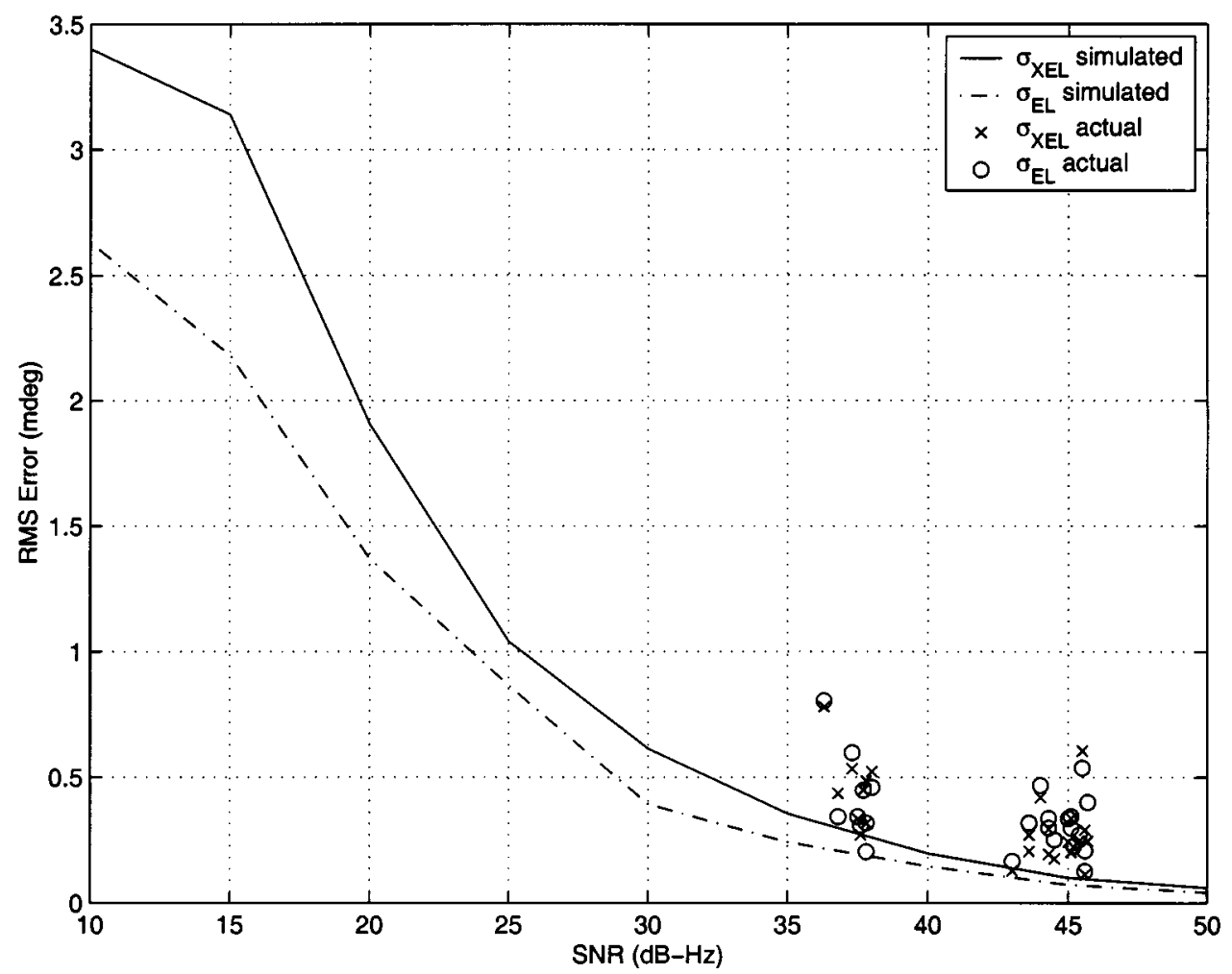

Fig. 22. Measured rms errors in $X E L$ and $E L$ versus simulation errors. The measurements were carried out at the DSS-14 station, while tracking the Ka-band downlink from the Cassini spacecraft.

issued every 15 s. Figs. 16 and 17 illustrate the RBF network's ability to recover from intentionally applied pointing errors in both $X E L$ and $E L$. These tracks, which covered antenna elevations ranging from $62.9^{\circ}$ down to $59.2^{\circ}$ with the antenna descending, have very short steady-state portions since the emphasis was on verifying the ability of the RBF network to deal with relatively large errors that may arise in the course of routine tracking. This is, in effect, an evaluation of the network's "acquisition" performance. The RBF network was able to correct intentionally applied pointing offsets, quickly pointing the antenna toward its nominal "on-source" direction. In Fig. 17, we see an example of the sharp climb in SNR that results from the pointing correction applied by the RBF network.

On "day 195," a different methodology was used. Inputs to the RBF network were the result of 1-s integration, and the network outputs were not averaged, but a delay of several seconds was applied, resulting in 6-s updates. No error offsets had been applied, but the track lasted about ten minutes, allowing observations of stability to be made. Total rms pointing errors remained $0.34 \mathrm{mdeg}$ or less throughout, and central and combined channel SNR remained stable as shown in Figs. 18 and 19.

Interpolated least squares tracks were also performed, yielding very similar results. Figs. 20 and 21 illustrate this algorithm's ability to correct intentionally applied pointing offsets. As with the day 68 RBF tracks, we see the system is able to correct intentionally applied pointing errors, thus restoring the antenna to its nominal "on-source" pointing direction. The recovery of lost SNR is readily apparent. It should be noted that in space communications the loss of one decibel can significantly increase the cost of a mission, making accurate pointing a high priority.
A summary of measured error standard deviations is presented in Fig. 22. This figure shows simulated rms errors over region 1 as defined in Table $\mathrm{I}$, which is just the origin $(0,0)$ in $(X E L, E L)$ space. Previous figures focused on region 3 . We note that the simulation curves provide a lower bound for the actual error standard deviations. Since the simulations do not model others causes of error, such as small amplitude vibrations due to imperfections in the gears, and buffeting by light wind, etc., it is expected that real-world rms errors will be somewhat higher than those obtained in simulation. However, we note that these errors lie well within the DSN pointing requirement of $0.8 \mathrm{mdeg}$. Similar results were obtained for the interpolated least squares algorithm. We also point out that the simulated curve for region 1 was generated only for the $45^{\circ}$ case, while these data were taken at elevations ranging from $37^{\circ}$ to $61^{\circ}$, so some discrepancies are unavoidable.

\section{CONCLUSION}

We have shown that both interpolated least squares and adaptive RBF networks, working in conjunction with an array feed compensation system, can point a 70-m deep space antenna with rms errors of $0.1-0.5 \mathrm{mdeg}$, under a wide range of signal-to-noise-ratios and antenna elevations, achieving significantly higher accuracies than the 0.8-mdeg benchmark for communications at Ka-band frequencies of $32 \mathrm{GHz}$. As such they demonstrate clear potential for highly accurate pointing of the 70-m DSN antennas, which is a challenging problem due to a multitude of time-varying distortions imposed on the antenna structure on a continuous basis. 
Results indicate that RBF networks perform as well as the quadratic interpolated least squares algorithms at varying antenna elevations for tracking and acquisition, and under a wide range of SNR conditions. At high antenna elevations, the quadratic interpolation method deteriorates for any significant offset from the "on-source" direction, making RBF networks especially attractive during high elevation operations.

Long-term adaptation of our algorithms for distorted antennas has also been shown to result in significantly improved performance. We have shown that adaptation of the linear combiner weights in the RBF network yields excellent performance for simple stretch, shrink, and translational distortions.

The system described in this paper has been tested while tracking the Cassini spacecraft using the 70-m antenna at Goldstone, CA. Very accurate pointing has been achieved, with total rms pointing errors often below $0.5 \mathrm{mdeg}$ and sometimes as small as $0.3 \mathrm{mdeg}$, particularly under high SNR conditions. This provides strong evidence that RBF networks and interpolated least squares algorithms both have significant potential for achieving accurate and robust antenna pointing control for Ka-band communications.

Future research should focus on improved adaptation strategies based on the use of power gradients for the purpose of adaptation. This has significant potential, and the authors hope to use it in conjunction with a "modified-LMS" or "modified pseudoinverse" strategy to update RBF combining weights, allowing the system to adapt to slow changes in the antenna. Additionally, there are plans to use both RBF networks and interpolated least squares algorithms to extract higher order information from the complex voltage data. Such information can be used to control adaptive RF optics systems to further reduce SNR losses and improve operating efficiency. Preliminary simulation work on these strategies is presently in progress.

\section{REFERENCES}

[1] V. A. Vilnrotter, E. R. Rodemich, and S. J. Dolinar Jr., "Real-time combining of residual carrier array signals using ML weight estimates," IEEE Trans. Commun., vol. 40, pp. 604-615, Mar. 1992.

[2] V. A. Vilnrotter, "Channel assignments for improved gain in baseband array feed compensation systems," IEEE Trans. Commun., vol. 42, pp. 2127-2133, May 1994.

[3] V. Vilnrotter and D. Fort. (1999, Nov.) Demonstration and Evaluation of the Ka-Band Array Feed Compensation System on the 70-Meter Antenna at DSS 14. [Online]. Available: http://tmo.jpl.nasa.gov.

[4] W. Lindsey and M. Simon, Telecommunication Systems Engineering. New York: Dover, 1973.

[5] R. Mukai, P. Arabshahi, and V. Vilnrotter, "An array feed radial basis function tracking system for NASA's deep space network antennas," presented at the Proc. Int. Joint Conf. Neural Networks, July 24-27, 2000.

[6] R. Mukai, V. Vilnrotter, P. Arabshahi, and V. Jamnejad. (2000, May) Computationally Intelligent Array Feed Tracking Algorithms for Large DSN Antennas. [Online]. Available: http://tmo.jpl.nasa.gov.

[7] S. Haykin, Neural Networks: A Comprehensive Foundation. Upper Saddle River, NJ: Prentice-Hall, 1998.

[8] S. Chen, C. F. N. Cowan, and P. M. Grant, "Orthogonal least squares learning algorithm for radial basis function networks," IEEE Trans. Neural Networks, vol. 2, pp. 302-309, Mar. 1999.

[9] P. Arabshahi, J. J. Choi, R. J. Marks II, and T. P. Caudell, "Fuzzy parameter adaptation in optimization: Some neural net training examples," IEEE Comput. Sci. Eng., vol. 3, no. 1, pp. 57-65, Spr. 1996.

[10] C. L. Lawson and R. J. Hanson, Solving Least Squares Problems. Philadelphia, PA: Soc. Ind. Appl. Math., 1995.

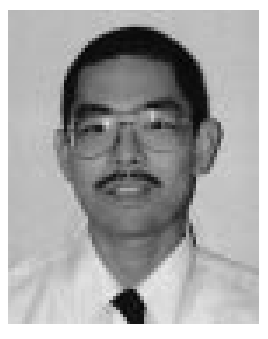

Ryan Mukai was born in San Dimas, CA. He received the B.S. degree in electrical engineering summa cum laude in 1997 and the M.S. in electrical engineering the same year, both from the University of California, Los Angeles. He is pursuing the Ph.D. degree at the same university.

Since 1999, he has been with the Jet Propulsion Laboratory, California Institute of Technology, Pasadena, as an academic part-time Researcher. $\mathrm{He}$ has successfully designed and implemented a working prototype of the interpolated least squares and RBF network tracking algorithms on the 70-m antenna at Goldstone, CA. His research interests include the application of neural networks to the detection of channel operating conditions in optical communication systems and the use of biologically inspired swarm intelligence algorithms in solving optimization problems.

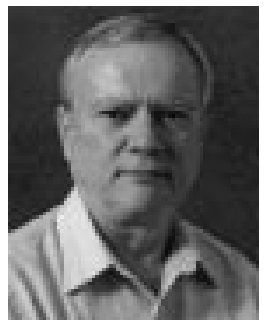

Victor A. Vilnrotter (M'79-SM'02) was born in Hungary in 1944. He received the B.S.E.E. degree from New York University, New York, with honors in 1971, the M.S. and E.E. degrees in electrical engineering from the Massachusetts Institute of Technology (MIT), Cambridge, in 1974 and, with the help of a Hughes Ph.D. Fellowship, the Ph.D. degree in electrical engineering and communications theory from the University of Southern California, Los Angeles, in 1978.

At MIT, he contributed to the development of an experimental over-the-horizon optical communication system operating between Lincoln Laboratory and the MIT campus. During his fellowship at the Hughes Aircraft Company, El Segundo, CA, he contributed to the development of wideband optical modulators using traveling-wave cavities, and helped develop and demonstrate coherent optical communications systems. He joined the Jet Propulsion Laboratory, California Institute of Technology, Pasadena, in 1979, where he is a Senior Engineer in the Digital Signal Processing Research group. He is currently conducting research on various topics in deep-space communications, including real-time electronic compensation for gravity and wind induced deformation of large antennas with focal-plane arrays, adaptive algorithms for optimum combining and tracking of spacecraft with large arrays, improved optical communications through atmospheric turbulence, and the application of quantum communications theory to topics in deep-space communications. He has authored or coauthored more than 80 articles in refereed journals, conference papers, and JPL publications, and has received numerous NASA awards for technical innovations.

Dr. Vilnrotter is a member of Eta Kappa Nu, Tau Beta Pi, Sigma Xi, and is a biographee in Marquis Who's Who in Science and Engineering.

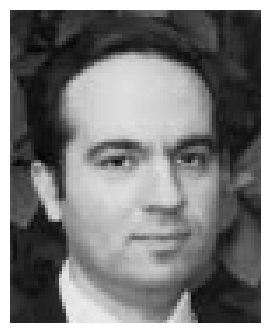

Payman Arabshahi (S'93-M'95) was born in Tehran, Iran, in 1968. He received the B.S.E. degree in electrical engineering from the University of Alabama, Huntsville, in 1988, and the M.S. and $\mathrm{Ph} . \mathrm{D}$. degrees in electrical engineering from the University of Washington, Seattle, in 1990 and 1994, respectively.

From 1994 to 1996, he was with the Department of Electrical and Computer Engineering, University of Alabama, Huntsville. From 1996 to 1997, he was with the Department of Electrical Engineering, University of Washington, and Neda Communications, Bellevue, Washington, where he continued his consulting work started in 1993. Since 1997, he has been with the Jet Propulsion Laboratory, California Institute of Technology, Pasadena, CA. He currently also serves on the faculty of the Department of Electrical Engineering, California Institute of Technology. His research interests are in swarm intelligence, adaptive and intelligent systems, digital signal processing, and bio-inspired communications. He is currently leading two tasks on dynamic signal processing technologies for optical communications, and biologically inspired routing and bandwidth allocation for sensor and satellite networks at JPL. He has more than 25 publications.

Dr. Arabshahi has been a Guest Editor of the IEEE TRANSACTIONS ON NEURAL NETWORKS; served as the Editor of the IEEE Neural Network Council's web page and newsletter; chaired the 1997 IEEE Computational Intelligence in Financial Engineering Conference, and helped with organization of ICASSP'98 and the IEEE Joint Conference on Neural Networks, July 2000. 


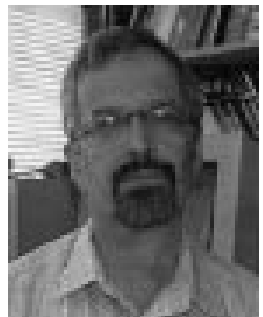

Vahraz Jamnejad (M'79-SM'99) received the Ph.D. degree in electrical engineering from the University of Illinois, Urbana-Champaign, in 1975, specializing in electromagnetics and antennas.

From 1975 to 1978, he was an Assistant Professor of electrical engineering at the Technical University of Tehran and from 1978-1979, he was a Visiting Professor at the University of Illinois. He joined the Jet Propulsion Laboratory of California Institute of Technology in 1979, where he has been engaged in research, software and hardware development, in various areas of spacecraft and ground antenna technologies and satellite communication systems. Among other things, he has been involved in the study, design, and development of ground and spacecraft antennas for future generations of Land Mobile Satellite Systems at L-band, Personal Access Satellite Systems at $\mathrm{K} / \mathrm{Ka}-\mathrm{band}$, as well as feed arrays and reflectors for future planetary missions. His later work on the communication satellite systems involved the development of ground mobile antennas for the $\mathrm{K} / \mathrm{Ka}$-band mobile terminals, for use with the ACTS satellite system. More recently, he has been in charge of research in new ultra low-loss lightweight ceramic waveguides, low-loss high-power S-band filters for the DSN ground antennas, analysis of submillimeter telescopes for the Planck project, and design of a beam waveguide telescope at millimeter and submillimeter range for the MIRO project. Over the past few years, he has also been active in research in parallel computational electromagnetics. Presently he is involved in developing antennas for MARS sample return mission and in studies on the applicability of large arrays of small aperture antennas for the NASA Deep Space Network. Over the years, he has received many United States patents and NASA awards.

Dr. Janmejad is a member of Sigma Xi and Phi Kappa Phi. 